\title{
ANALYSIS OF THE sU-leuA LOCUS IN SALMONELLA TYPHIMURIUM
}

\author{
G. W. P. DAWSON and P. F. SMITH-KEARY \\ Department of Genetics, Trinity College, Dublin
}

\section{INTRODUCTION}

Received 6.vii.6o

WE have been studying a leucine auxotroph of Salmonella typhimurium, designated leu-15I, which when plated on minimal medium spontaneously reverts to wild type and to a type which, in the absence of leucine, grows more slowly than the wild type. Those slow growing reversions have been shown to be due to an additional mutation at a locus linked to the leucine locus. (Smith-Keary 1960). This second mutation partially suppresses the requirement for leucine, and it has been given the symbol su-leuA. Thus su-leuA-I, su-leuA-2 etc. stand for independently isolated suppressors of leu-I $5 \mathrm{I}$.

A locus that controls the ability to ferment arabinose $(a r a B)$ is known from transduction experiments to be linked to leu. We have previously shown that su-leuA is linked to leu and that the order is su-leuA, leu, araB (Smith-Keary, 1960). These linkages permit the genetic analysis of independently isolated su-leuA mutants and it is possible to show that these mutants specifically suppress $l e u-\mathrm{I} 5^{\mathrm{I}}$ and not some other leu mutants and that they are mutations at different sites in the su-leuA locus. We have been able to deduce the relevant linkage map of the su-leuA region.

\section{MATERIALS AND METHODS}

The wild type and all the mutants we used were of the LT 2 strain of Salmonella typhimurium. Genetic material was transduced with the temperate phage PLT 22, or when sensitive transductants were required, with the $\mathrm{H}_{4}$ mutant of this phage.

All stocks of leu-15I also carried $m e A-22$ (requires methionine) and $\operatorname{try} B-2$ (requires tryptophane). In all the relevant experiments adequate amounts of methionine and tryptophane were added to the media and in the following account we shall omit references to these genes and to these requirements.

The mutants leu-8, leu-10, leu-20, leu-32, leu-38, leu-39 and araB-9 were obtained from the Carnegie Department of Genetics, Cold Spring Harbor, Long Island, New York.

The preparation of the phage, the method of performing the transductions and the media we used have been previously described (Smith-Keary, 1960). In this account we use the following abbreviations for the different media:

\footnotetext{
MM minimal medium.

EMM minimal medium enriched with broth.

$\mathrm{MM}+\mathrm{L} \quad$ media supplemented with 0.002 per cent. dl-leucine.

$\mathrm{EMM}(\mathrm{arab})+\mathrm{LL}$ EMM containing $0 \cdot 2$ per cent. arabinose in place of glucose and supplemented with $0 \cdot 004$ per cent. dl-leucine.

EMB eosin-methylene blue agar.
} 
We characterised the colonies obtained in transduction experiments in one of two ways. When selection was made for colonies that did not require leucine, this was confirmed by streaking or plating each colony on $M M$ and $M M+L$, and their ability to ferment arabinose was determined by streaking on EMB. When selection was for those colonies that could ferment arabinose, the colonies were first streaked on EMB and, from each streak, a colony that fermented arabinose was streaked or plated on $\mathrm{MM}$ and on $\mathrm{MM}+\mathrm{L}$ to determine whether it required leucine. This sequential streaking is necessary to isolate pure $\mathrm{ara}^{+}$clones from the transductant colonies as these are often a mixture of $\mathrm{ara}^{+}$and $\mathrm{ara}^{-}$cells.

\section{EXPERIMENTAL ANALYSIS}

Before we describe the genetic analysis of the suppressor locus it is necessary to develop the argument of the previous paper in which the linkage order su-leuA leu-I 5 I leu-39 araB-9 was established so as to decide whether $l e u-\mathrm{I} 5 \mathrm{I}$ falls within or without the known limits of the leu locus and also whether the su-leuA sites are outside or inside these limits. If the su-leuA sites are within the leu locus we are probably investigating complementation within a locus; if outside we are probably investigating two separate loci.

\section{(i) Order of leu sites}

It is known that leu-39 is near the end of the leu locus nearest to $a r a B$ and that $l e u-32$ is near the end furthest from $a r a B$. We know that $l e u$ - $5_{5}$ I is further from $a r a B$ than $l e u-39$ and we now have to show that it lies between leu-32 and leu-39. As neither leu-32 nor leu-I $5^{\mathrm{I}}$ is available in combination with an $\operatorname{ara} B$ marker the most satisfactory tests (transducing either leu- $5_{5}$ I $^{a r a B} B^{-}$with phage raised on leu-32 $a r a B^{+}$or leu-32 $a r a B^{-}$with phage raised on leu-I $5^{\text {I }} a r a B^{+}$, selecting for leu ${ }^{+}$ transductants and scoring for $\mathrm{ara}^{+}$and $\mathrm{ara}^{-}$) cannot be made. Instead we used the following methods.

(a) Transductions between leu-I $5 \mathrm{I}$, leu- 32 and leu-39. The frequency of prototrophs from transductions between leu-32, leu-I 5 I and leu-39 in all possible combinations are set out in table $\mathrm{I}$. That the frequency obtained from the transduction leu-39 $(X) l e u-32$ is higher than from the transduction $l e u-39(\times)$ leu-I $5^{\mathrm{I}}$ indicates that $l e u-\mathrm{I} 5^{\mathrm{I}}$ is probably located between leu-32 and leu-39. The only comparison inconsistent with this conclusion is the higher frequency of prototrophs from the transduction $l e u-\mathrm{I}_{5} \mathrm{I}(X)$ leu-39 than from the transduction leu-32 $(\times)$ leu-39. This inconsistency is probably attributable to the greater effectiveness of leu-I 5 I than leu- 32 as recipient. This greater effectiveness is clearly shown when transductions are made with phage grown on a wild type strain (table $\mathrm{I}$, last column).

(b) Transductions between araB-9 and leu-32, leu-15 $\mathrm{I}$ and leu-39. Phage grown on each of the leucine mutants was used to infect araB-9. $\mathrm{ara}^{+}$transductants were selected by plating on $\operatorname{EMM}(\mathrm{arab})+\mathrm{LL}$ and these were then tested for their ability to grow in the presence and absence of leucine. The results are set out in table 2. The results of reciprocal transductions, selecting for leucine independence and 
testing the transductants for their ability to ferment arabinose, are set out in table 3 .

TABLE I

Wild type transductants per $10^{8}$ survivors in reciprocal transductions between different leu auxotrophs

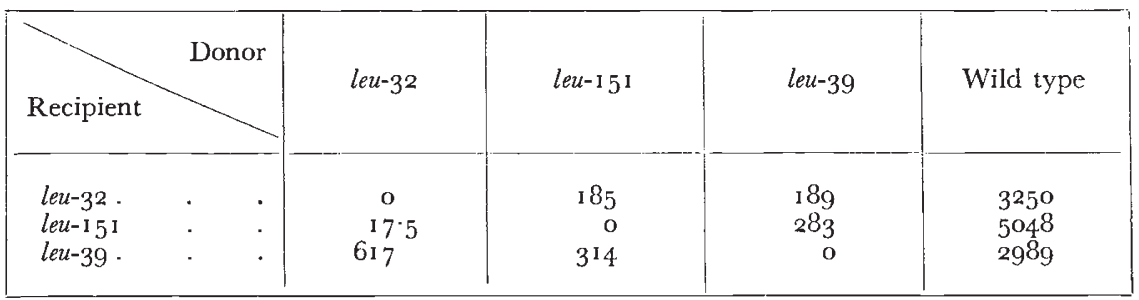

TABLE 2

Relative frequencies of $\mathrm{leu}^{+}$and $\mathrm{leu}^{-}$transductants from the transductions araB-9 $(\times)$leu-32, araB-9 $(X)$ leu-I $5 \mathrm{I}$ and araB-9 $(X)$ leu-39, selecting for ara ${ }^{+}$transductants

\begin{tabular}{|c|c|c|c|}
\hline & \multicolumn{3}{|c|}{ Donor } \\
\hline & $l e u-32$ & $l e u-15^{1}$ & leu-39 \\
\hline $\begin{array}{l}l e u^{+}: \\
\text {leu- } \\
\text { Total : }\end{array}$ & $\begin{array}{r}148 \\
76 \\
224\end{array}$ & $\begin{array}{r}100 \\
68 \\
168\end{array}$ & $\begin{array}{r}35 \\
81 \\
116\end{array}$ \\
\hline Per cent. leu- & $33 \cdot 93$ & $4^{0} \cdot 4^{8}$ & $69 \cdot 83$ \\
\hline
\end{tabular}

TABLE 3

Relative frequencies of $\mathrm{ara}^{+}$and $\mathrm{ara}^{-}$transductants from the transductions leu- $32(\times)$ araB- 9 , leu-I5I (X) araB-9 and leu-39 (X) araB-9, selecting for leu ${ }^{+}$transductants

\begin{tabular}{|c|c|c|c|}
\hline & \multicolumn{3}{|c|}{ Recipient } \\
\hline & leu-32 & $l e u-151$ & leu-39 \\
\hline 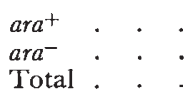 & $\begin{array}{l}228 \\
135 \\
363\end{array}$ & $\begin{array}{l}162 \\
100 \\
262\end{array}$ & $\begin{array}{l}145 \\
260 \\
405\end{array}$ \\
\hline Per cent. $\operatorname{ar} a^{-}$ & $37 \cdot 19$ & $38 \cdot 17$ & $64 \cdot 20$ \\
\hline
\end{tabular}

In both series of experiments the frequency of joint transduction of the selected and unselected marker (per cent. leu- in table 2, per cent. ara $a^{-}$in table 3) was highest with leu-39, lowest with leu-32 and intermediate with leu-I5I. As the frequency of joint transduction usually decreases with increase in the distance between the markers, though not necessarily proportionately, these results confirm the position of leu-I5I between leu-32 and leu-39. Further confirmation 
of the relative positions of $l e u-32$ and leu- $\mathrm{I}_{5} \mathrm{I}$ is provided by the results of the transductions described in section $3.2(b)$.

Recently Margolin has sent us representative mutants of the 4 complementation groups which he has discovered within the leu locus: leu-12 I, leu-129, leu-126 and leu-128. Margolin has shown that these complementation groups correspond to the linear arrangement of the sites (Margolin, I959) and that their linkage order is (personal communication):

\begin{tabular}{|c|c|c|c|c|}
\hline I 2 I & 129 & I 26 & 128 & $\operatorname{araB}$ \\
\hline$=$ & $\underbrace{2}$ & $=$ & $=$ & \\
\hline I & II & III & IV & \\
\hline
\end{tabular}

From the results of the transduction leu-12 I $\operatorname{araB}-9(\times) l e u-\mathrm{I} 5 \mathrm{I}$ and its reciprocal we have found that leu-I2I is nearer $a r a B$ than is leu-I5I. Thus the order of these sites and leu-32 is leu-32, leu-15 I, leu-1 $2 \mathrm{I}$.

No abortive transductions occurred when leu-121, as recipient, was transduced with either leu-I5I or leu-32, but these did occur when $l e u-\mathrm{I} 29$ or wild type was used as the donor. We conclude that $l e u-\mathrm{I} 5 \mathrm{I}$ and $l e u-32$ are in the same complementation group as leu-121. This is consistent with the linkage data and suggests that there is unlikely to be an additional complementation group to the left of Margolin's group I.

\section{(ii) su-leuA and leu as separate but linked loci}

We have already shown that the first isolated suppressor of leu-I 5 I (su-leuA-I) is linked to leu and $a r a B$. By the following two series of experiments five other independently isolated suppressors have been shown to be linked, and no suppressor of $l e u-15 \mathrm{I}$ has been found which is not linked to leu and $a r a B$.

(a) Linkage of suppressors with leu and araB. Phage grown on each of the six suppressed mutants (su-leuA-I leu-I5I, su-leuA-2 leu-I5I etc.) was used to infect leu-39 araB-9 and selection made for leucine independent transductants. Most of these transductants were slow growing. These slow growing colonies were tested for their ability to ferment arabinose and the frequency of joint transduction of su-leuA-x leu-I $5^{\mathrm{I}}$ and $a \mathrm{raB}^{+}$showed non-significant variation between 53.I per cent. and $57 \cdot 2$ per cent. with the different suppressors. Significant variation is not expected as this figure measures the relative frequencies of cross-overs in regions $a$ and $b$ and in regions $a$ and $c$ and this is independent of the position of the su-leuA site.

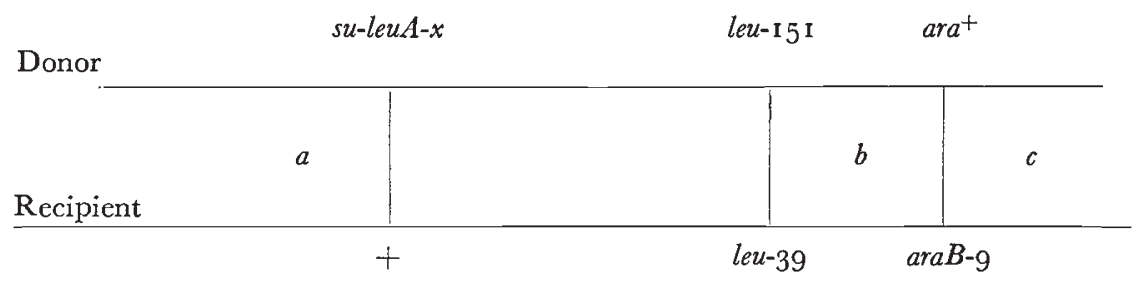


These results show that the suppressor site in each of the six suppressed mutants is linked to the leu and $\operatorname{araB}$ loci.

(b) su-leuA and leu as separate loci. Four different suppressed mutants were used as donors in transductions with $\operatorname{araB}-9$ as recipient. $\mathrm{ara}^{+}$transductants were selected and then characterised for their ability to grow in the absence of leucine. These transductants must arise, ignoring quadruple cross-overs, by one crossover in region $a$ and the second in region $b$ (wild type for leucine) or in region $c$ (auxotroph for leucine) or in region $d$ (suppressed mutant-grows slowly in absence of leucine).

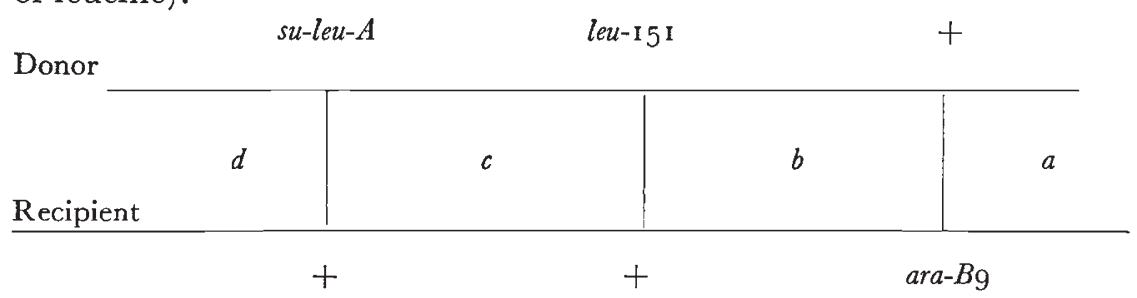

As all three classes can be detected, the results (table 4) provide an estimate of linkage of su-leuA with araB-9. These results give an average of 45.28 per cent. joint transductions of leu-15 I and araB-9 and an average of 43.67 per cent. joint transductions of su-leuA and

\section{TABLE 4}

Relative frequencies of fast growing, slow growing and leucine auxotroph transductants from the transduction araB- $9(\times)$ suleuA-x leu-15 $\mathrm{I}$ araB ${ }^{+}$using different su-leuA mutants, selecting for ara ${ }^{+}$transductants

\begin{tabular}{|c|c|c|c|c|c|c|c|}
\hline \multirow{2}{*}{$\begin{array}{c}\text { su-leuA } \\
\text { allele of } \\
\text { donor }(x)\end{array}$} & \multirow{2}{*}{$\begin{array}{l}\mathrm{ara}^{+} \\
\text {clones } \\
\text { tested }\end{array}$} & \multicolumn{2}{|c|}{ Fast } & \multicolumn{2}{|c|}{ Slow } & \multicolumn{2}{|c|}{ Auxotroph } \\
\hline & & Observed & Per cent. & Observed & Per cent. & Observed & Per cent. \\
\hline $\begin{array}{l}\text { I } \\
4 \\
2 \\
6\end{array}$ & $\begin{array}{r}108 \\
331 \\
214 \\
89\end{array}$ & $\begin{array}{r}63 \\
\text { I } 84 \\
\text { I } 13 \\
46\end{array}$ & $\begin{array}{l}58 \cdot 33 \\
55 \cdot 59 \\
52 \cdot 80 \\
51 \cdot 69\end{array}$ & $\begin{array}{r}45 \\
\text { I } 39 \\
98 \\
4^{2}\end{array}$ & $\begin{array}{l}4 I \cdot 67 \\
4 I \cdot 99 \\
45 \cdot 79 \\
47 \cdot 19\end{array}$ & $\begin{array}{l}0 \\
8 \\
3 \\
\text { I }\end{array}$ & $\begin{array}{l}O \\
2 \cdot 42 \\
I \cdot 40 \\
I \cdot 12\end{array}$ \\
\hline Totals & 742 & 406 & $54 \cdot 72$ & 324 & $43 \cdot 67$ & I 2 & $I \cdot 62$ \\
\hline
\end{tabular}

araB-9. The suppressor locus is thus quite close to leu-I5I. Because of this close linkage it is necessary to consider the possibility that the suppressor sites are within the limits of the leucine locus.

Table 2 shows that there is 33.93 per cent. joint transduction of leu-32 with araB-9, but, while it is reasonable to take this with other evidence as showing that the order is leu-32 leu-I5I $\operatorname{araB}$ the data are not sufficiently extensive to decide whether leu-32 is relatively far from or very close indeed to leu-I $5 \mathrm{I}$. This latter possibility is indicated 
by the similarity in the percentage of joint transductions of leu- 32 and $a r a B-9$ and of $l e u-15$ I and $a r a B-9$ when selection is for $l e u^{+}$(table 3).

The best available evidence that the suppressor locus is beyond the known limits of the leucine locus, that is further from araB than leu-32, is from the transduction su-leuA-I leu-I $5^{\mathrm{I}} \operatorname{araB}-9(X)$ leu-32 using $\mathrm{H}_{4}$ phage and selecting for $\mathrm{rra}^{+}$transductants. If the order is su-leuA-I $l e u-32$ leu-15 $5^{\text {I }}$ araB-9 this transduction can be represented as follows:

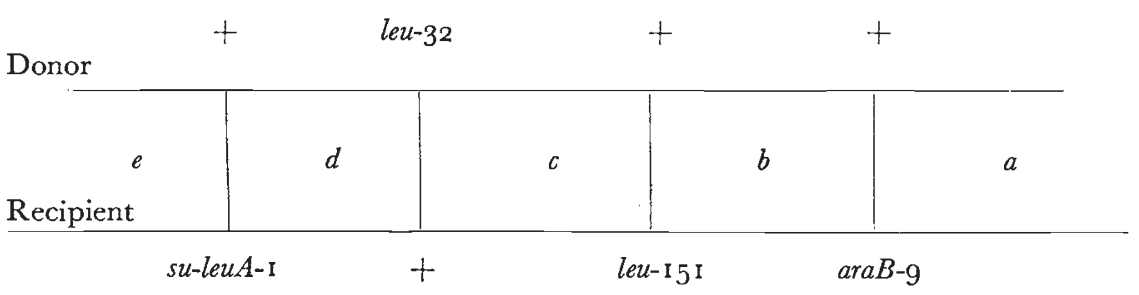

Crossovers in regions $a$ and $b$ will give su-leuA-1 leu-15 5 colonies. Crossovers in regions $a$ and $c$ will give su-leuA-I leu+ colonies.

Crossovers in regions $a$ and $d$ will give su-leuA-I leu-32 leu-I5I colonies.

Crossovers in regions $a$ and $e$ will give su-leuA $A^{+} l e u-32$.

If the order is leu-32 su-leuA-I leu-15 I $a r a B-9$ this transduction can be represented as follows:

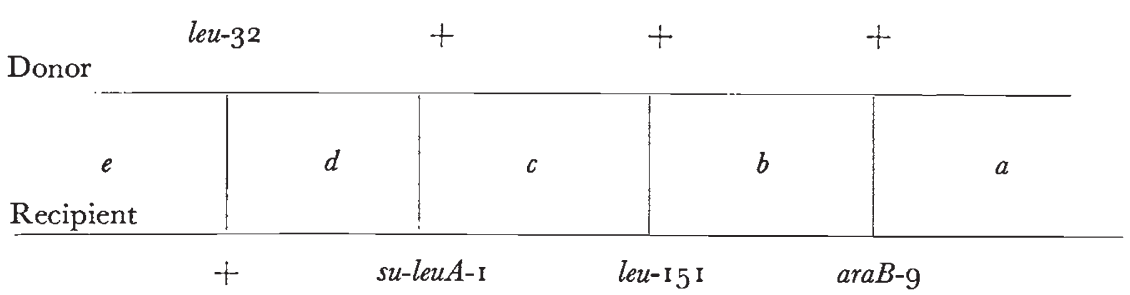

Crossovers in regions $a$ and $b$ will give su-leuA-I leu-I 5 I colonies.

Crossovers in regions $a$ and $c$ will give su-leuA-I leu+ colonies.

Crossovers in regions $a$ and $d$ will give su-leuA+leut colonies.

Crossovers in regions $a$ and $e$ will give su-leuA$A^{+} l e u-32$ colonies.

Thus, omitting genotypes arising by quadruple crossovers, all the colonies which grow as wild type in the absence of leucine will, if the first order is correct, be su-leuA-I leu+; if the second order is correct they will be either $s u$-leuA-I leu+, or, su-leuA ${ }^{+} l e u^{+}$. Whether both these genotypes arise can be investigated by taking phage sensitive transductants that grow like wild type in the absence of leucine, raising phage PLT 22 on them, and using this phage to infect leu-I5I. If such a transductant is $s u$-leuA-I leu both " fast" and "slow" colonies will be produced; five such transductants were scored in this way and all were su-leuA-I leu+. Two transductants from a similar cross involving su-leuA-4, one from a cross involving su-leuA-5 and one from a cross involving su-leuA-2 were also found to be $s u$-leuA-4 (or 5 or 2) leu $u^{+}$. 
We conclude that while there remains the slight possibility that su-leuA is between leu-32 and leu-I5 I (being much closer to leu-32 than to $l e u$-I 5 I) it is more likely that su-leuA is outside the known limits of the leu locus. This likelihood is increased by the discovery that one of the suppressors (su-leuA-I) is a multisite mutant (see next section) and it would seem unlikely that a single site mutant in the leucine locus could be suppressed by such a major disturbance within the same complementation group of the locus.

These transductions are further evidence for the relative positions of $l e u-32$ and $l e u-\mathrm{I} 5 \mathrm{I}$. If $l e u-32$ were nearer to $a r a B$ than $l e u-\mathrm{I} 5 \mathrm{I}$, the initial transductants would be unlikely to carry the suppressor mutation and no slow growing colonies would arise in the second transduction with $l e u$-I $5 \mathrm{I}$ as recipient.

\section{(iii) Linkage order of the suppressor sites}

If the independently isolated suppressed mutants arise by mutations at different sites within the $s u$-leuA locus it should be possible to plot the linkage order of these sites. Consider a transduction su-leuA-x leu-I $5 \mathrm{I}$ $\operatorname{araB}-9(\times)$ su-leuA-y leu-I $5 \mathrm{I}$ where $x$ and $y$ are mutations at different sites of the su-leuA locus. If $y$ is distal to $x$ it should be possible to discover leucine auxotrophs among the $\mathrm{ara}^{+}$transductants: these are unlikely to be discovered if $y$ is proximal to $x$.

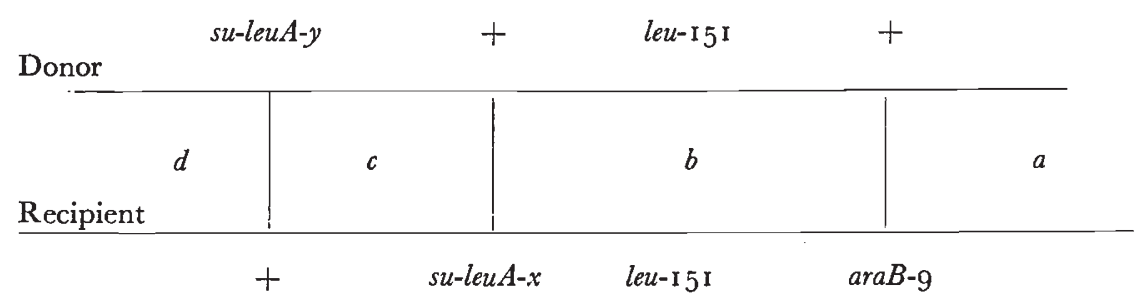

Those $a r a^{+}$transductants that arise by crossovers in $a$ and $c$ will be leucine auxotrophs.

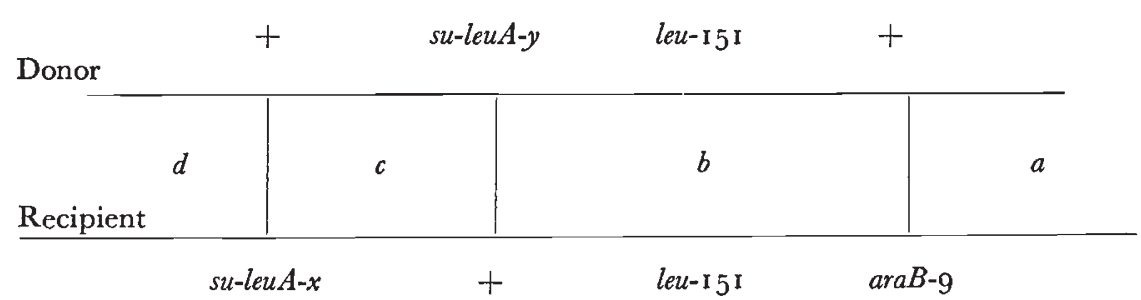

Only those very rare transductants that arise by quadruple crossovers in $a$ and $b$, and in $c$ add $d$ will be leucine auxotrophs.

Such an analysis of the six independently isolated suppressed mutants requires that each of these su-leuA-x leu-I5I genotypes be introduced into the $a r a B-9$ stock. This was done by transducing 
su-leuA-x leu-15 I into leu-39 araB-9 with $\mathrm{H}_{4}$ phage and selecting for transductants that grow in the absence of leucine. From among these phage sensitive, arabinose non-fermenting colonies that only grow slowly in the absence of leucine were chosen. Since su-leuA-x does not suppress leu-39 (see next section) the strains obtained must be su-leuA-x leu-15I araB-9.

The proportions of leucine auxotrophs that were found among the $\mathrm{ara}^{+}$transductants when the six suppressed mutants were crossed in all possible ways are set out in table 5 . It is conspicuous, and in

\section{TABLE 5}

su-leuA-x leu-I5I araB-9 $(\times)$ su-leuA-y leu-15I, selecting for ara ${ }^{+}$transductants. Number of leucine auxotrophs among ara ${ }^{+}$transductants tested

\begin{tabular}{|c|c|c|c|c|c|c|c|}
\hline & & \multicolumn{6}{|c|}{$s u$-leuA allele in donor (su-leuA-y) } \\
\hline & & I & 2 & 3 & 4 & 5 & 6 \\
\hline \multirow{6}{*}{$\begin{array}{l}\text { su-leuA } \\
\text { allele in } \\
\text { recipient } \\
(\text { su-leuA-x })\end{array}$} & I & $0 / 100$ & $0 / 58$ & $0 / 80$ & $0 / 90$ & $0 / 92$ & $0 / 110$ \\
\hline & 2 & $0 / 92$ & $0 / 75$ & $0 / 75$ & I/104 & $0 / 105$ & $0 / 73$ \\
\hline & 3 & $0 / 92$ & I $/ 64$ & $0 / 60$ & $2 / 30$ & $\mathrm{I} / \mathrm{I} 43$ & $0 / 80$ \\
\hline & 4 & $0 / 103$ & $0 / 70$ & $0 / 60$ & $0 / 60$ & $0 / 30$ & $0 / 60$ \\
\hline & 5 & $0 / 94$ & $2 / 82$ & o/I 53 & $8 / 144$ & $0 / 30$ & $4 / 192$ \\
\hline & 6 & $0 / 9 \mathrm{I}$ & I $/ 68$ & $0 / 192$ & $4 / 90$ & $0 / 160$ & $0 / 130$ \\
\hline
\end{tabular}

accord with the above argument, that auxotrophs are never found in both of the two possible transductions involving any two suppressed mutants.

su-leuA-I differs from the other suppressed mutants in yielding no auxotrophs in any of the crosses. We conclude that this is a multisite mutant (Demerec et al., I956) extending over the whole distance marked by the mutant sites of the other suppressed mutants. If this were interpreted as a deletion extending close to the end of the leu locus it would explain the absence of auxotrophs from the transduction araB-9 (X) su-leuA-I leu-I 5I (table 4).

Excluding su-leuA-I and transductions with homologous phage we can make the following deductions of the order of the su-leuA sites, with reference to $\operatorname{araB}$, from each of the crosses:

$\begin{array}{cccccc}\begin{array}{c}\text { su-leuA } \\ \text { donor }\end{array} & \begin{array}{c}\text { su-leuA } \\ \text { recipient }\end{array} & \begin{array}{c}\text { Deduced } \\ \text { order }\end{array} & \begin{array}{c}\text { su-leuA } \\ \text { donor }\end{array} & \begin{array}{c}\text { su-leuA } \\ \text { recipient }\end{array} & \begin{array}{c}\text { Deduced } \\ \text { order }\end{array} \\ 2 & 3 & 2-3 \text {-araB } & 4 & 5 & 4-5 \text {-araB } \\ 2 & 4 & 4-2 \text {-araB } & 4 & 6 & 4-6 \text {-araB } \\ 2 & 5 & 5-2 \text {-araB } & 5 & 2 & 2-5 \text {-araB } \\ 2 & 6 & 2-6 \text {-araB } & 5 & 3 & 5-3 \text {-araB } \\ 3 & 2 & 2-3 \text {-araB } & 5 & 4 & 4-5 \text {-araB } \\ 3 & 4 & 4-3 \text {-araB } & 5 & 6 & 6 \text {-5-araB } \\ 3 & 5 & 5-3 \text {-araB } & 6 & 2 & \text { 2-6-araB } \\ 3 & 6 & 6-3 \text {-araB } & 6 & 3 & \text { 4-6-araB } \\ 4 & 2 & 4-2 \text {-araB } & 6 & 4 & 6 \text {-araB } \\ 4 & 3 & 4-3 \text {-araB } & 6 & 5 & \end{array}$


With the exception of the transduction where su-leuA-6 was in the donor and su-leuA-3 in the recipient, from which about two auxotrophs were expected and none found, all the results agree with the order of the sites being:

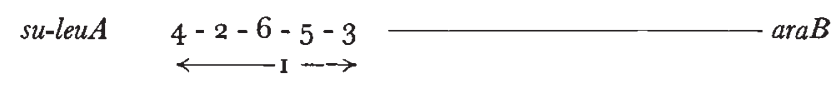

This order is in full agreement with the crude estimates of the degree of linkage of su-leuA-4, su-leuA-2 and su-leuA-6 with araB previously obtained (table 4 ).

The proportion of auxotrophs from the transduction su-leuA-x leu-I I I $\operatorname{araB}-9(X)$ su-leuA-y leu-I 5 I (table 5), which is a rough measure of the distances between the su-leuA sites, and the proportion of auxotrophs from the transductions araB-9 $(X)$ su-leuA-x leu-I5I (table 4), which is a rough measure of the distances between the su-leuA sites and leu-I $5^{\mathrm{I}}$, are of the same order of magnitude. Thus su-leuA and leu are probably adjacent loci, and site $\mathrm{I}_{5} \mathrm{I}$ is close to the end of the leu locus nearest the su-leuA locus, but not quite as close as leu-32.

\section{(iv) Specificity of suppressors}

We have tested three of the suppressors to discover whether they will suppress other leucine mutants than leu-I 5 I. The three suppressors tested were su-leuA-I, 2 and 5 , and the leucine mutants were leu-32, I5 I, I 2 I, 8, I0, 20, 38 and 39. Of these leucine mutants the first three are known to be in the same complementation group; we do not know the complementation groups of the other leucine mutants.

Phage PLT 22 grown on each of 3 stocks separately carrying the 3 mutant suppressors in combination with $l e u^{+}$(obtained from the experiments described in section 3 (ii) (b)) was used to infect each of the leucine mutants. Leucine independent transductants were selected and these were examined to discover whether they included any which had slow growth in the absence of leucine. If slow growing transductants were not found we concluded that the su-leuA mutant site did not suppress that particular leucine mutant. Slow growing transductants were recovered only when leu-I 5 I was infected.

su-leuA-I, 2 and 5 therefore specifically suppress $l e u$ - I 5 I and do not even suppress other mutant sites in the same complementation group as $l e u-I 5$.

\section{NOTE ON METHODOLOGY}

We know very little about many of the factors that influence the outcome of a transduction experiment with Salmonella typhimurium. For example we are not certain whether all transduced bacterial genetic fragments that include a particular locus are of identical length. The use of some strains as recipient inexplicably results in a larger number of transductants than the use of other strains that are very similar 
genetically. We know too little about the degree of negative interference between crossovers to be able to take satisfactory account of this in our predictions. A transduced fragment of bacterial genome may undergo a number of successive incorporations in the recipient cell and its descendants. These complications make us wary of reaching decisive conclusions from particular experiments. For example, the experiments described in section $\mathrm{I}$ indicate that $l e u-32$ is further from araB than is $l e u-15 \mathrm{I}$ but they do not rule out the possibility that the reverse is true. The additional evidence in section 3 (ii) (b), makes the reverse order more unlikely. We can only conclude that, on the available evidence the order leu-32 leu-I $5^{\mathrm{I}}$ araB is the most likely. All our conclusions are simply the most probable explanations of the data, after ignoring complications that we have no means of assessing.

We would emphasise two technical problems in the present study. First, when leucine-independent transductants are selected by plating on media without leucine, the distinction between slow growing (suppressed mutants) and fast growing (wild type) transductants cannot be made on the transduction plates. Each colony must be picked off and replated on media without leucine so as to give isolated colonies. This involves considerable labour and accounts for some of the data not being more extensive than they are. Second, it is known that a transduced fragment carrying $a r a B$ may undergo successive incorporations in the recipient cell and its descendants. Thus when we select for transduction of $\mathrm{leu}^{+}$and then streak out the colonies to score for arabinose fermentation some may be scored $\mathrm{ara}^{+}$which, if it had been possible to score only the result of the incorporation that introduced the $l e u^{+}$, would have been scored $\mathrm{ara}^{-}$. There may therefore be in many experiments an excessive proportion of $\mathrm{ara}^{+}$and a deficiency of $\mathrm{ara}^{-}$. There is, however, no good reason for supposing that this bias varies significantly from experiment to experiment, as we used the same arabinose marker in all the experiments.

\section{DISCUSSION}

The su-leuA, leu and $a r a B$ factors are linked and this has permitted a genetical analysis of the su-leuA locus. We have shown that independently isolated suppressed mutants of $l e u$ - $5^{1} \mathrm{I}$ arise by mutations at different sites within the suppressor locus. It seems that any of a number of mutations, even a multisite mutation, within su-leuA will specifically suppress $l e u-\mathrm{I} 5 \mathrm{I}$ and will not suppress seven other leu mutants, two of which are in the same complementation group as $l e u$-I 5I. It might be thought possible formally to restate these conclusions in terms of complementation and not suppression and say that many mutational changes within su-leuA complement leu-r $5 \mathrm{I}$. However the term "complementation" is usually reserved for a situation where a wild type phenotype only occurs when either both or neither mutant sites are present, and not when only one is present, 
and here the single mutant su-leuA leu+ is not an auxotroph but has a nearly wild type phenotype, su-leuA leu-151 an intermediate phenotype, and only su-leuA+leu-1 $5^{1}$ is an auxotroph. While it is convenient to maintain this distinction between "suppression " and "complementation" it is by no means certain that the biochemical basis of these phenomena will necessarily always be of a different type. That su-leuA and leu are probably adjacent regions is perhaps a hint that the biochemical basis of this suppression may be similar to those mechanisms which are invoked to explain complementation within a functional unit. Thus su-leuA may be part of the same functional unit as the leu locus, having the property that at least some of the possible mutational changes within it only slightly inhibit leucine synthesis and complement a small minority of mutations within the leu part of the unit.

Among other microorganisms site specific suppressors are known in Neurospora (Giles and Partridge, 1953; Yanofsky and Bonner, I955) and Aspergillus (Käfer, I958), but in these and other previous studies a genetic analysis has either not been possible or has not been attempted. It may well be that site specific suppression will be found that has its basis in mutation at a single site in a suppressor locus, but the present study has demonstrated that here at least, such suppression arises by many different site, and multisite, mutations within the one locus.

\section{SUMMARY}

I. Slow growing reversions of leu-15 $5^{\mathrm{I}}$ have been shown to be due to mutations at a suppressor locus, su-leuA, linked to leu and araB.

2. su-leuA is probably adjacent to and does not overlap the leu locus.

3. Five separate sites within the su-leuA locus have been plotted. su-leuA-I is probably a multisite mutant.

4. su-leuA-I, 2 and 5 suppress leu-I 5 I but do not suppress leu-8, ro, 20, 32, 38, 39 or 121 .

5. A linkage map of the su-leuA leu araB region has been constructed and the probable order of the markers shown to be:

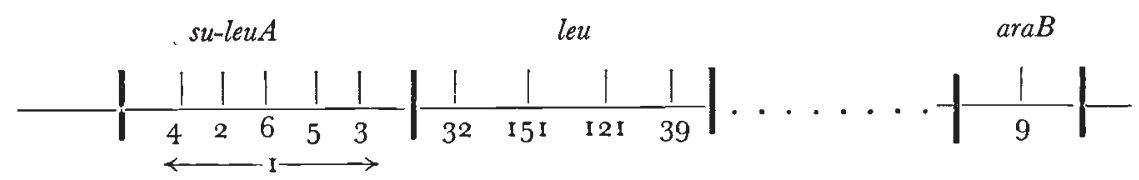

Acknowledgments.-The research reported herein has been sponsored in part by the Office, Chief of Research and Development, U.S. Department of the Army, through its European Research Office, and in part by the Medical Research Council of Ireland.

\section{REFERENCES}

Demerec, M., Moser, H., ClOWES, R. C., LAHR, E. L., OZEKI, H., AND VIelmetTer, W. 1956. Bacterial Genetics. Carnegie Inst. Wash. Year Book, 55, 301-315. 
GILes, N. H., AND PARTRIDGe, C. W. H. 1953. The effect of a suppressor on allelic inositolless mutants in Neurospora crassa. P.N.A.S., 39, 479-488.

KÄFER, E. 1958. An 8-chromosome map of Aspergillus nidulans. Advances in Genetics, 9, I05-145.

MARGOLIN, P. 1959. Linkage order and complementation among non-identical alleles of the leucine locus in Salmonella typhimurium. Genetics, 44, 525-526 (abstract).

SMrth-KEARY, P. F. 1960. A suppressor of leucineless in Salmonella typhimurium. Heredity, ${ }_{4}, 6 \mathrm{I}-7 \mathrm{r}$.

YANOFSKY, C. AND BONNER, D. M. 1955. Non-allelic suppressor genes affecting a single $t d$ allele. Genetics, 40, 602-603 (abstract). 